\title{
Probing Dark Energy through Scale Dependence
}

\author{
Mariele Motta, ${ }^{1,2}$ Ignacy Sawicki, ${ }^{1}$ Ippocratis D. Saltas ${ }^{3}$ Luca Amendola, ${ }^{1}$ and Martin Kunz ${ }^{4,5}$ \\ ${ }^{1}$ ITP, Ruprecht-Karls-Universität Heidelberg, Philosophenweg 16, 69120 Heidelberg, Germany \\ ${ }^{2}$ Instituto de Física Gleb Wataghin - UNICAMP, 13083-970 Campinas, SP, Brazil \\ ${ }^{3}$ School of Physics \&6 Astronomy, University of Nottingham, Nottingham, NG7 2RD, United Kingdom \\ ${ }^{4}$ Département de Physique Théorique and Center for Astroparticle Physics, \\ Université de Genève, Quai E. Ansermet 24, CH-1211 Genève 4, Switzerland \\ ${ }^{5}$ African Institute for Mathematical Sciences, 6 Melrose Road, Muizenberg, 7945, South Africa
}

\begin{abstract}
We consider the consequences of having no prior knowledge of the true dark energy model for the interpretation of cosmological observations. The magnitude of redshift-space distortions and weaklensing shear is determined by the metric on the geodesics of which galaxies and light propagate. We show that, given precise enough observations, we can use these data to completely reconstruct the metric on our past lightcone and therefore to measure the scale- and time-dependence of the anisotropic stress and the evolution of the gravitational potentials in a model-independent manner. Since both dark matter and dark energy affect the visible sector only through the gravitational field they produce, they are inseparable without a model for dark energy: galaxy bias cannot be measured and therefore the distribution of dark matter determined; the peculiar velocity of dark matter can be identified with that of the galaxies only when the equivalence principle holds. Given these limitations, we show how one can nonetheless build tests for classes of dark energy models which depend on making measurements at multiple scales at a particular redshift. They are null tests on the model-independent observables, do not require modeling evolution in time and do not require any parametrization of the free functions of these models - such as the sound speed. We show that one in principle could rule out or constrain the whole class of the most-general scalar-tensor theories even without assuming the quasi-static limit.
\end{abstract}

\section{INTRODUCTION}

The introduction of dark energy relaxes many of the assumptions behind $\Lambda$ CDM cosmology. Not only can the background evolution be altered, but in many models the dark energy is predicted to have significant perturbations. As a result, the simple $\Lambda$ CDM relations between the dark matter perturbations and the gravitational fields sourced by them are no longer valid. The detection of a form of dark energy different from a cosmological constant hinges on the unambiguous determination of how these relations are broken.

Various approaches to this goal have been taken. On the level of background evolution, null tests for the compatibility of the cosmic expansion history with $\Lambda \mathrm{CDM}$ were proposed in Refs $[1,2]$, while a principal-component analysis of the equation of state constraints was developed in Ref. [3]. On the level of linear perturbations, parametrizations are usually used to give a model-independent description of the growth of largescale structure [4-9], although principal component analysis has also been performed [10] (see [11] for a short review). Alternatively one may try to focus on understanding the limits that a general-relativity-like theory must obey, but describing the remaining freedom with a parametrization framework [12-14] or attempt to describe the properties of dark energy through an effective framework [15-18]. Such approaches, when contrasted with data, usually require parametrizations in order to break down degeneracies, but which simultaneously introduce parametrization-dependent biases.

Our approach, introduced in Ref. [19], has been to take a step back and ask what the observables which we can in principle extract from cosmological probes are when we do not assume a particular model for dark energy. We have argued that as a result of the presence of unknown dark energy perturbations, the only modelindependent observables are ratios of quantities typically considered to be unambiguous measurements. This implies that such variables as the dark-matter growth rate $f$ or dark-matter perturbation amplitude $\sigma_{8}$ are not actually observable in a model-independent way.

In this paper, we elucidate further this framework, showing that a helpful way to understand our previous conclusions is to realize that our cosmological probes (galaxy counts and weak lensing) rely on tracers which propagate on geodesics and therefore can at best determine the gravitational potentials which affect their motion. Indeed, we show that given sufficient data one could in principle map out the whole metric on our past lightcone. Therefore the slip parameter describing anisotropic stress is a direct observable not requiring any parametrization. Moreover, since it is a ratio of two variables containing the same stochastic source, itself it is free of cosmic variance. On the other hand, what we cannot do at all is to ever probe the dark matter distribution using these means, since the dark matter does not interact directly with our probes, only by sourcing the gravitational potential. This means the effective Newton's constant, which is important to quantify dark energy perturbations, cannot be observed. Given this difficulty, we nonetheless describe a strategy for constraining dark energy models, generalizing our work away from the quasi-static limit considered previously. 
We start in section II by defining the notation, and reviewing the assumptions and the model-independent observables that we have found before. We then demonstrate in section III that the metric and the gravitational slip can indeed be reconstructed without further assumptions, but that the effective Newton's constant is not fully determined. In section IV we illustrate the practical consequences of this result by devising consistency tests for different classes of models. For general dark energy models in the quasi-static limit and dynamical Horndeski models with anisotropic stress, we are able to formulate general constraints that relate different scales at fixed redshifts and that can be tested by observations without the need to parametrize any free functions. For models that have no anisotropic stress, we propose a slightly more complicated procedure using $\mathrm{k}$-essence as a worked example. We are again able to test whether this model class is compatible with the data, and if it is, a recipe allows one to reconstruct the model's free functions without parametrization. We summarize the definitions of the variables used and the status of their observability in table I located on page 11.

\section{ASSUMPTIONS AND NOTATION}

In Ref. [19], we investigated which properties of the constituents of the late universe are observable without making prior assumptions as to the model of dark energy. We started off with a minimal set of assumptions, which we require to be able to interpret our observations at all:

- The geometry of the universe is well described by scalar linear perturbations in a FriedmannLemaître-Robertson-Walker metric with scale factor $a(t)$,

$$
\mathrm{d} s^{2}=-(1+2 \Psi) \mathrm{d} t^{2}+a^{2}(t)(1+2 \Phi) \mathrm{d} \boldsymbol{x}^{2} .
$$

We mostly neglect spatial curvature, but since it is observable, its existence would not change the principal results of this paper. The parameter $\Omega_{k 0}$ would enter some of the equations, but given how small it seems to be, it would not affect them in any significant way.

- The matter content is pressureless. We do not differentiate between dark matter and baryons. We neglect radiation. We assume that the equivalence principle holds and therefore that both these components follow geodesics of the same metric. This allows us to assume that there is no velocity bias between dark matter and galaxies, although we will comment on what breaking this assumption would mean.

- The galaxy distribution is related to the matter distribution through a potentially time- and scale-dependent deterministic linear bias, $\delta_{\text {gal }}=$ $b(k, a) \delta_{\mathrm{m}}$. We make no assumption about any particular form of the bias function.

We have then assumed the idealized situation in which we can perform current and future cosmological measurements to as good an accuracy as is required for the range of scales and redshifts we are attempting to characterize: supernova luminosities, the distribution of galaxies, baryon acoustic oscillation measurements and weak lensing shear.

We have argued that without an a priori assumption of a particular model or parametrization for dark energy, there is no way of knowing the evolution or even the initial conditions for the dark energy distribution. Indeed, one of the aims of late-universe cosmology is exactly to measure this, both on a background and perturbation level.

We shall use the standard notation to describe the evolution of the cosmological background

$$
E(z)^{2}=\Omega_{\mathrm{m} 0}(1+z)^{3}+\Omega_{k 0}(1+z)^{2}+\Omega_{X},
$$

with $E(z) \equiv H(z) / H_{0}$, the dimensionless Hubble parameter, $\Omega_{\mathrm{m} 0}$ the contribution of matter to the total density, $\Omega_{X}$ the contribution of dark energy and $\Omega_{k 0}$ the spatial curvature.

For the purpose of perturbations, we can describe the solution realized by the Universe using a number of ratios of the gravitational potentials in the metric $-\Phi$ and $\Psi$ - and the dark matter configuration - the density contrast $\delta_{\mathrm{m}}$ and its peculiar velocity divergence $\theta_{\mathrm{m}}$. In all of the following, we denote with perturbation variables (e.g. $\Phi, \Psi, \delta_{\mathrm{m}}$, etc.) the positive-definite square root of their power spectrum, as is common in the literature. We will always be working in Fourier space and primes will denote a derivative with respect to $\ln a$.

In particular, we define ratios of the perturbation variables: the slip parameter $\eta$, the effective dimensionless Newton's constant $Y$, and the dark-matter growth rate $f$, which are all in principle arbitrary functions of scale and redshift

$$
\begin{aligned}
& \eta(k, z) \equiv-\Phi / \Psi, \quad Y(k, z) \equiv-\frac{2 k^{2} \Psi}{3 \Omega_{\mathrm{m}} \delta_{\mathrm{m}}}, \\
& f(k, z) \equiv-\frac{\theta_{\mathrm{m}}}{H \delta_{\mathrm{m}}} \approx \frac{\delta_{\mathrm{m}}^{\prime}}{\delta_{\mathrm{m}}}
\end{aligned}
$$

where the scale $k$ has been expressed in units of the cosmological horizon, $k \equiv k_{\text {com }} / a H$ and the prime denotes a derivative with respect to $\ln a$. The perturbation variables are subject to cosmic variance which will always put a limit on how precisely their measurement reflects the actual variance. However, provided that there is no additional source of stochastic perturbations in the redshift range of interest, their ratios for a single mode $k$ are in fact free of cosmic variance (similar to the argument in Ref. [20]). It is these ratios, independent of the initial conditions, that we will use as variables testing dark energy. 
Our definition for $f$ is a little unusual, but it reduces to the standard one sufficiently subhorizon, whenever the gravitational potentials are slowly varying on the scales in question, since by the continuity equation,

$$
\delta_{\mathrm{m}}^{\prime}+H^{-1} \theta_{\mathrm{m}}=-3 \Phi^{\prime} .
$$

We can also think of $Y$ as describing the size of the dark energy perturbation relative to the one in matter, although adjusted for anisotropic stress; at subhorizon scales and assuming no correlation between matter and dark energy fluctuations,

$$
\eta Y \approx 1+\frac{\Omega_{X} \delta_{X}}{\Omega_{\mathrm{m}} \delta_{\mathrm{m}}} .
$$

One can form other ratios of all these variables, which are not independent. In particular, a ratio useful for us will be

$$
\varpi \equiv \frac{f}{\Omega_{\mathrm{m} 0} Y}=\frac{3(1+z)^{3} \theta_{\mathrm{m}}}{2 H E^{2} k^{2} \Psi}
$$

One should always bear in mind that these ratios in principle describe the particular solution realized by our Universe rather than necessarily representing fundamental properties of the dark energy. Both $Y$ and $\eta$ are unity on sub-horizon scales in $\Lambda$ CDM, while $f \approx \Omega_{\mathrm{m}}^{0.55}$ in the concordance model. All of these variables are scale independent subhorizon in $\Lambda$ CDM. This is not the case for most dark energy models.

It is important to stress that even measuring all these variables does not give all the information on the configuration of dark energy. In principle, there could be many internal degrees of freedom which do not have an effect on the gravitational field produced (essentially isocurvature modes).

In Ref. [19], our focus was on determining the properties of dark energy by attempting to extract from observations its impact on dark matter. We first recovered the result of Ref. [21], demonstrating that no combination of geometrical probes of background evolution can ever split the contribution to total energy density of the universe of matter and dark energy. These probes can at best recover the Hubble parameter as a function of redshift up to an overall normalization, $E(z)$, and independently measure any spatial curvature $\Omega_{k 0}$. Such properties as the dark energy equation-of-state parameter, $w$, or $\Omega_{\mathrm{m} 0}$ are not observable, but can only be obtained by making assumptions on the form of $w$ or performing a particular parametrization. This degeneracy has been dubbed the dark degeneracy. Measurements of the local expansion rate $H_{0}$ have been performed [22]. However, they are sensitive to the local cosmic variance and therefore do not necessarily reflect the averaged expansion rate today [23].

We then extended this study to perturbations. We have demonstrated that weak lensing and galaxy distribution observations can be reduced to a measurement of three variables as function of scale and redshift

$$
\begin{aligned}
& A=G b \delta_{\mathrm{m}, 0}, \quad R=G f \delta_{\mathrm{m}, 0}, \\
& L=\Omega_{\mathrm{m} 0} G Y(1+\eta) \delta_{\mathrm{m}, 0},
\end{aligned}
$$

where $G$ is the growth function normalized to one today, $\delta_{\mathrm{m}, 0}$ is the scale-dependent density contrast of dark matter. In Ref. [19] we used the notation $\delta_{\mathrm{m}, 0}=\sigma_{8} \delta_{\mathrm{t}, 0}$ to make explicit the fact that there is an unknown distribution of dark energy perturbations present which affects gravitational measurements. As we have previously argued, the amplitude of the density contrast today depends on the whole history of the common evolution of the unknown dark energy and dark matter, and the initial conditions in both these components. It is thus impossible to measure $\delta_{\mathrm{m}, 0}$ without a knowledge of the bias $b$ : the scale-dependence of $\delta_{\mathrm{m}, 0}$ is unknown without a knowledge of the dark energy model and there are no measurements of the amplitude $\sigma_{8}$ that would be independent of the DE model (see the discussion in section V). Thus we have argued that the only model-independent observables are ratios of the measurements (7):

$$
\begin{aligned}
& P_{1}=\beta=R / A=f / b, \\
& P_{2}=E_{G}=L / R=\Omega_{\mathrm{m} 0} Y(1+\eta) / f, \\
& P_{3}=R^{\prime} / R=f+f^{\prime} / f,
\end{aligned}
$$

where for $P_{1}$ and $P_{2}$ we have provided also the notation more frequently used by the community [24]. It is possible to measure each of these observables as a function of scale and redshift. Any other such model-independent observable can be re-expressed as a combination of the above and their derivatives. Indeed, a combination that appears frequently is

$$
\frac{L^{\prime}}{L}=P_{3}+\frac{P_{2}^{\prime}}{P_{2}}
$$

which is not independent of the others. We will refer to the observables $P_{i}$ as the primary model-independent observables. The observable $P_{1}$ contains the bias which cannot be measured in a model-independent manner and therefore we will not use it any further in our discussion (see the discussion of measurability of the bias in section V). We should stress that these results, for example, imply that the growth rate of dark matter $f$ cannot be measured without picking a particular model or at least a parametrization for dark energy.

We will now approach the question of observability slightly differently than above, in a way that may be somewhat more physical. We will show that what is actually observable in a model-independent manner are the gravitational potentials, i.e. the metric. What is not possible to do using the observations we can perform is to split the sources of the curvature into a dark matter and dark energy part. 


\section{DIRECTLY OBSERVABLE PROPERTIES}

We are going to begin this section by demonstrating that using weak lensing (WL) and redshift-space distortions (RSD) we can in principle reconstruct the metric in which the galaxies and light propagate as a function of redshift and scale.

Weak lensing is a direct probe of the lensing potential

$$
k^{2} \Phi_{\text {lens }}=k^{2}(\Psi-\Phi)=-\frac{3(1+z)^{3}}{2 E^{2}} L .
$$

Thus a tomographic weak lensing map should really be thought of as a map of the lensing potential as a function of redshift and scale [25]. It is not actually a direct measurement of the underlying distribution of dark matter, which can only be extracted by accounting for the unknown perturbations of dark energy also contributing to the potential.

The above statement is quite natural and unsurprising. We will now show that RSD can be thought of in the same way. Traditionally one maps the velocities of the galaxy field onto the underlying dark-matter distribution through the bias and relates the RSD to the dark matter growth rate. ${ }^{1}$ This is not necessary, however. Fundamentally, the Kaiser formula [26] for the redshift-space galaxy density $\delta_{\text {gal }}^{z}$ is a statement about a correction to the real-space galaxy number density $\delta_{\text {gal }}$ resulting from the peculiar velocities of the galaxies $\theta_{\text {gal }}[27$,

$$
\delta_{\text {gal }}^{z}(k, z, \mu)=\delta_{\text {gal }}(k, z)-\mu^{2} \frac{\theta_{\text {gal }}(k, z)}{H},
$$

with $\mu$ the direction cosine. Now, rather than relating this velocity to dark matter evolution, we can make a choice that allows us to avoid any assumption about the velocity bias: the galaxies are test particles propagating on geodesics of the metric, whatever the actual source of the curvature,

$$
\left(a^{2} \theta_{\text {gal }}\right)^{\prime}=a^{2} H k^{2} \Psi
$$

with $k \equiv k_{\text {com }} / a H$. We can now integrate, neglecting the integration constant, to discover that from the angular dependence of the two-dimensional galaxy power spectrum we can extract,

$$
A=\delta_{\text {gal }}, \quad R=-\frac{\theta_{\text {gal }}}{H}=-\left(a^{2} H\right)^{-1} \int a^{2} H k^{2} \Psi \mathrm{d} \ln a .
$$

This approach allows us to see that linear RSD are really a measurement of the gravitational potential $\Psi$ which accelerates the galaxies

$$
-k^{2} \Psi=R^{\prime}+R\left(2+\frac{E^{\prime}}{E}\right) .
$$

\footnotetext{
${ }^{1}$ See e.g. [26, 27]. This is how we proceeded in Ref. [19].
}

Since all the terms on the r.h.s. of Eq. (14) are observables, we can use RSD to measure the gravitational potential as a function of redshift and scale. Combining this with the information from the lensing (10), allows us in principle to map out the metric on our past light cone. This procedure will break down on scales where the galaxies are no longer propagating on geodesics, i.e. where interactions become significant or non-linearities of the metric invalidate the linear approximation. In principle, one should extend this simple estimate for relativistic effects which will grow in importance closer to the horizon, as in Refs [28-32].

In models where both the baryons and the dark matter feel the same forces (in particular, not in e.g. coupled quintessence [33]), we can assume that there is no velocity bias between galaxies and matter, thus $\theta_{\text {gal }}=\theta_{\mathrm{m}}$. Through the dark-matter continuity equation (4), we can recover the standard result (7) that $R=\delta_{\mathrm{m}}^{\prime}$ for sufficiently slowly varying potentials, which was the basis of our discussion in [19]. When this assumption is relaxed, which implies a violation of the equivalence principle, $\theta_{\mathrm{m}} / H_{0}$ is no longer an observable because of the a priori unknown velocity bias. It will then no longer be true that RSD directly give the derivative of the dark matter density contrast, $\delta_{\mathrm{m}}^{\prime}$.

We will now attempt to characterize the dark energy, which will require us to return to the split between the dark matter and the dark energy. Indeed, the total information that could potentially be extracted from gravitybased experiments on the dark energy configuration realized in our Universe is encoded in the ratios (3). We will now show that there is a fundamental difference in our ability to extract the slip parameter $\eta$ from observations and the effective Newton's constant, $Y$ or the growth rate $f$.

Firstly, since through Eqs (10) and (14) we can obtain the potentials, we can directly measure the slip parameter by taking the appropriate ratio

$$
\eta=-\Phi / \Psi=\frac{3 P_{2}(1+z)^{3}}{2 E^{2}\left(P_{3}+2+\frac{E^{\prime}}{E}\right)}-1
$$

This is the same result as we obtained in Ref. [19]. However, in the current approach, we have not had to make any assumptions on the behavior of dark matter, or even its existence. Given sufficiently precise measurements of the galaxy distribution and weak lensing covering common redshifts and scales, the slip parameter can be measured redshift by redshift, scale by scale, without having to depend on a better- or worse-motivated parametrization and constraints that are obtained through a total integrated signal.

Another result that will be useful in what follows is that, since the potentials are observable, their evolution can also be measured. We can define an observable akin to the growth rate for DM, the evolution rate for the 
Newtonian potential,

$$
\begin{aligned}
\Gamma \equiv \frac{\Psi^{\prime}}{\Psi} & =\frac{L^{\prime}}{L}-\frac{\eta^{\prime}}{1+\eta}-1 \\
\frac{\Phi^{\prime}}{\Phi} & =\Gamma+\frac{\eta^{\prime}}{\eta}=\frac{L^{\prime}}{L}+\frac{\eta^{\prime}}{\eta(1+\eta)}-1
\end{aligned}
$$

or equivalently, the evolution of $\Psi$ can obtained purely from RSDs in terms of $P_{3}$ by differentiating Eq. (14). Moreover, it is also possible to measure the variable $\varpi$ defined in Eq. (6)

$$
\varpi=\frac{1+\eta}{P_{2}}
$$

Again, the observability of $\varpi$ essentially depends on there being no equivalence principle violation, since otherwise we do not have access to $\theta_{\mathrm{m}}$.

The measurement of anisotropic stress is a very important discriminator between various classes of dark energy models. However, in order to characterize the configuration of dark energy in our universe, we also have to map the effective Newton's constant, $Y$, which describes the relative size of the energy-density perturbations of dark energy and matter, Eq. (5). Unfortunately, it is impossible to construct a combination of observables (8) which would allow one to obtain a model-independent measurement of $Y$. This is a result of the fact that there exists no cosmological probe which would be able to determine the dark matter density $\delta_{\mathrm{m}}$ without a prior assumption of a particular dark energy model. As we have argued above, both lensing and RSDs probe the metric, with RSDs at best providing a measurement of the derivative $\delta_{\mathrm{m}}^{\prime}$, provided the potentials vary sufficiently slowly and the equivalence principle be not violated. The best that we can do is to differentiate the definition of $Y$, Eq. (3), to obtain the differential equation

$$
\frac{Y^{\prime}}{Y}+\Omega_{\mathrm{m} 0} \varpi Y=1+\Gamma .
$$

where we have dropped a term related to $\Phi^{\prime}$ irrelevant subhorizon. The coefficients in Eq. (18) are observables (assuming the equivalent principle), but we have only succeeded in obtaining a first-order differential equation for $\Omega_{\mathrm{m} 0} Y$. Given measurements of observables (8), equation (18) can be integrated. However, an initial condition must be supplied, effectively the value of $\delta_{\mathrm{m}}$ at the initial time, which is not an observable.

The above limitation is again a result of the dark degeneracy, here appearing on the level of perturbations. There is no gravity-based experiment which could tell us in what part the local gravitational potential is sourced by matter and in what part by dark energy. In principle, one could hope to measure the mass density of dark matter particles by using information from direct and indirect detection experiments. However, since no experiment can currently determine the physical density of dark matter on the scales of interest, the split between dark matter and dark energy is fundamentally arbitrary. We can always redefine the dark energy model to include a part of the dark matter. ${ }^{2}$

A part of the dark degeneracy is related to our lack of knowledge of $\Omega_{\mathrm{m} 0}$ and implies that $\Omega_{\mathrm{m} 0}$ can be absorbed into the definition of $Y$ through $Y \rightarrow \Omega_{\mathrm{m} 0} Y$. However, this is not sufficient to fully specify the split between dark matter and dark energy: we still have the freedom to supply an arbitrary initial condition for (18). For example, one could imagine a situation where at some particular redshift there are no dark energy perturbations but only those for matter, i.e. $Y=1$. Once this choice has been made, the observations in combination with Eq. (18) tell us how $Y$ evolves as a function of redshift. However, any initial choice is as good as any other. Eq. (18) essentially evolves the values of $Y$ to the next timeslice as a result of our assumption that the part of the universe that we have designated to be dark matter is pressureless and follows geodesics of a metric we have mapped.

As a result of this degeneracy, the best that we can do is to propose a behavior for $Y$, resulting from some sort of model with some free parameters, and then ask the question whether the observations are consistent with the evolution mandated by Eq. (18). Eq. (18) should therefore be thought of as a consistency relation for $Y$ which can be used to rule out particular models but not to measure this quantity. We will give an example of such a procedure in section IV.

The reason for this fundamental limitation is our lack of knowledge of the density bias. The measurement of the amplitude of the galaxy power spectrum only describes galaxy number counts; given a measurement of the bias for some particular tracer, the dark matter density contrast could also be reconstructed. Methods for the measurement of bias have been proposed and used, but they assume various properties of $\Lambda \mathrm{CDM}$ in a fundamental way. They cannot be used to constrain dark energy models departing from the cosmological constant in general (see section $\mathrm{V}$ for a discussion).

In light of the above results, we can reinterpret slightly the measurements that we are performing in $\Lambda$ CDM. The weak-lensing and galaxy-distribution observations are in fact sensitive to the perturbed geometry, since they involve tracers that propagate on geodesics. It is only because the cosmological constant is not perturbed that these potentials can be ascribed directly to dark matter and the standard language recovered. It should be intuitively clear that without the knowledge of dark energy perturbations we must

\footnotetext{
2 See for example Ref. [34] where a model with no dark matter, just dark energy, was proposed. The dark energy has sound speed $c_{\mathrm{s}}^{2}=0$ and can, for example, replicate exactly the phenomenology of the background and dark-matter linear perturbations in $\Lambda \mathrm{CDM}$.
} 
have arrived at the model-independent result we have described: only geometrical and not dynamical properties can be measured by tracers propagating on geodesics.

The overall conclusion is that without particular assumptions on the model of dark energy, cosmological observations allow at most for the mapping out of the dimensionless Hubble parameter $E(z)$ as a function of redshift and the two gravitational potentials as a function of both redshift and scale. No information about the relative distribution of dark energy and dark matter can be obtained without an assumption of a particular model or at least some parametrization.

\section{TESTING SCALAR-FIELD MODELS}

In this section, we will discuss our ability to use the observable properties described in section III to constrain or even exclude generalized scalar-tensor models of dark energy.

\section{A. Quasi-Static Dark Energy}

As we have already discussed in Ref. [19], perturbations in scalar-tensor dark energy models frequently can be approximated to evolve in the quasi-static approximation, where the dark energy follows the dark matter perturbations and the time derivatives are negligible (see also Ref. [35]). For the Horndeski Lagrangian [36, 37], which is the most general scalar-tensor theory involving no more than second derivatives, this limit was derived in Ref. [38]. This derivation assumes that the only scales relevant to the problem are the Jeans length (determined by the sound speed) and the Compton wavelength, determined by the effective mass of the scalar perturbations. Dispersion relations are in principle more complicated in these models, but apart from the mass, the sound speed does provide the smallest scale by the virtue of being defined in the $k \rightarrow \infty$ limit. Under the assumption that the speed of sound is close to that of light and the scales larger than the Jeans length lie outside of the scales probed by the observations, the effective Newton's constant and the slip parameter (3) in these general scalartensor models take the form

$$
Y=h_{1}\left(\frac{1+k^{2} h_{5}}{1+k^{2} h_{3}}\right), \quad \eta=h_{2}\left(\frac{1+k^{2} h_{4}}{1+k^{2} h_{5}}\right),
$$

where the functions $h_{i}$ are purely functions of redshift determined by the Lagrangian describing the dark energy model (see Ref. [19] for the explicit form of $h_{1-5}$ ).

The quasi-static limit is really the requirement that the dark energy perturbations follow the dark matter ones in a very constrained way. Dark matter evolves on the geodesics of the combined gravitational potential while the dark energy perturbation must follow the very precise prescription defined by Eqs (19), without any dynamics of its own.

One can use Eq. (15) to test the consistency of the observed universe with the slip parameter $\eta$ given by the form (19). As we have described in Ref. [19], given measurements of the observable (15) at more than three different scales per redshift, we can test whether the data are consistent with a description for $\eta$ of the form (19). If not, then the anisotropic stress cannot be described as a manifestation of a scalar-tensor theory in the quasi-static limit.

Since dark matter is supposed to move in a known way on geodesics of the metric and we can map this metric out, the consistency of the form of $Y$ given in the quasistatic limit can be tested, despite the non-observability described in section III. Inserting the form (19) into the consistency relation (18) allows one to ask, redshift by redshift, whether the quasi-static form for $Y$ is consistent with the observational data, i.e. whether the simple scale dependence for $Y$ driven by the coefficients $h_{i}$ is sufficient to explain the observations. As a result of taking the time derivative in (18), the number of free parameters increases to seven (including $\Omega_{\mathrm{m} 0}$ ), thus a larger number of measurements at each redshift is required to overconstrain the system, but it is in principle not any more difficult than for the anisotropic stress.

The main takeaway from this discussion is that if the quasi-static limit can be assumed, the dark energy configuration can be tested by looking for a particular scale dependence of observables redshift bin by redshift bin, rather than as a single integrated fit to the observations with a particular parametrization chosen for the functions $h_{i}$.

\section{B. Beyond the Quasi-Static Limit}

The quasi-static limit considered in section IV A is somewhat unsatisfactory, given that there are not necessarily a priori reasons why the DE should evolve so as to follow the DM perturbations so closely. $Y$ specifies the size of the dark energy density perturbation relative to the dark matter one. It should therefore be intuitively clear that on any single spatial hypersurface, one can in principle arrange the dark energy density in an arbitrary manner and therefore there can be no restriction on the form of $Y$ if the dark energy can be perturbed freely. By choosing the quasi-static limit, we give up this freedom, since all the relative velocities between DM and DE must vanish and the distribution of the DE is purely determined by the density perturbation of dark matter.

Nonetheless, once the initial conditions are specified, $Y$ will have to evolve in a manner consistent with the equation of motion for the scalar or, equivalently, the conservation of the DE energy-momentum tensor, whatever the model behind it. Since the metric is mapped out, and the dark matter propagates on its geodesics, it is possible to test whether the evolution of the dark en- 
ergy part is consistent with a particular class of models.

Another requirement arises in situations in which nonzero anisotropic stress is detected. Its measurement through Eq. (15) gives additional information on the distribution of the scalar-field perturbations. This then has to be consistent with the distribution implied by the effective Newton's constant $Y$ on that spatial slice. Theories with anisotropic stress are in general more complicated than those without and contain more parameters. However, in this way we can have an additional handle with which to constrain them.

We will now discuss a couple of examples to illustrate how one might go about constraining models using the model-independent variables.

\section{Consistency with k-essence}

Here, we will turn to the simplest class of scalar field models, those described by the Lagrangian $\mathcal{L}_{\phi}=K(\phi, X)$ [39-41]. The energy momentum tensor possessed by this kind of dark energy has perfect-fluid form and at linear level all the properties are described by the equation of state $w$ and the sound speed $c_{\mathrm{s}}$ [42]. This example will provide for sufficient complexity in order to demonstrate the logic of our method and the fundamental limitations of constraining the dark energy model space as a result of the non-observability of $Y$, while having the advantage of being familiar to a wide audience. Note that all uncoupled quintessence models are contained within this class as are perfect-fluid models, provided only scalar perturbations be considered.

We shall not give the full Einstein equations here, but it will suffice to say that the combined k-essence and dark matter energy-momentum tensor (EMT) depends on the perturbation variables schematically as

$$
\begin{aligned}
\delta T_{0}^{0} & \supset \delta \phi, \dot{\delta \phi}, \delta_{\mathrm{m}}, \\
\delta T_{i}^{0} & \supset \delta \phi, \theta_{\mathrm{m}}, \\
\delta T_{i}^{i} & \supset \delta \phi, \dot{\delta \phi}, \\
\delta T_{j}^{i}-1 / 3 \delta_{j}^{i} \delta T_{k}^{k} & =0,
\end{aligned}
$$

with $\delta \phi$ the perturbation of the k-essence scalar and $\delta_{\mathrm{m}}, \theta_{\mathrm{m}}$ describing the DM density contrast and the divergence of the peculiar velocity. This model does not allow for any anisotropic stress and therefore it could be immediately excluded if, even at just one redshift and scale, $\eta \neq 1$. We will assume that no such detection was made and therefore we will take $\Phi=-\Psi$. We can then use the combination of the Hamiltonian and momentum constraints [i.e. the $(00)$ and $(0 i)$ Einstein equations], to eliminate the scalar-field perturbations $\delta \phi$ and $\dot{\delta \phi}$ in the (ii) Einstein equation, obtaining the exact equation for the evolution of the gravitational potential in k-essence models in the presence of dark matter

$$
\begin{aligned}
\Psi^{\prime \prime} & +\left(4+\frac{E^{\prime}}{E}+3 c_{\mathrm{a}}^{2}\right) \Psi^{\prime}+\left(3+2 \frac{E^{\prime}}{E}+3 c_{\mathrm{a}}^{2}\right) \Psi+(21) \\
& +c_{\mathrm{s}}^{2} k^{2} \Psi=-\frac{3}{2} \Omega_{\mathrm{m}}\left(c_{\mathrm{s}}^{2} \delta_{\mathrm{m}}+3\left(c_{\mathrm{a}}^{2}-c_{\mathrm{s}}^{2}\right) k^{-2} H^{-1} \theta_{\mathrm{m}}\right) .
\end{aligned}
$$

where the adiabatic sound speed,

$$
c_{\mathrm{a}}^{2} \equiv \frac{\dot{p}_{X}}{\dot{\rho}_{X}}=-\frac{6 E^{\prime} / E+2\left(E^{\prime} / E\right)^{2}+2^{E^{\prime \prime}} / E}{9 \Omega_{\mathrm{m} 0} E^{-2}(1+z)^{3}+6^{E^{\prime}} / E},
$$

is fully determined by the observable expansion history up to $\Omega_{\mathrm{m} 0} . \theta_{\mathrm{m}} / H=R$ is of the order $k^{2} \Psi$ and is observable. Equation (21) is quite standard, see the closely related result in e.g. [43, Eq. 7.51]

Apart from the parameters of this model - the constant $\Omega_{\mathrm{m} 0}$ and the sound speed $c_{\mathrm{s}}$, which can be a function of time - all the quantities on the left-hand-side of Eq. (21) are observable. What is not observable is $\delta_{\mathrm{m}}$, as we have explained in section III. Since, at any one redshift slice, the dark-matter configuration can in principle be arbitrary, Eq. (21) does not by itself provide a constraint on the theory space. However, we can think of Eq. (21) as a measurement of $Y$ given the assumption that the dark energy model belongs to the k-essence class:

$$
\begin{aligned}
c_{\mathrm{s}}^{2} k^{2} \hat{Y}^{-1}= & \Gamma^{\prime}+\left(\Gamma+3+E^{\prime} / E+3 c_{\mathrm{a}}^{2}\right)(1+\Gamma)+ \\
& +E^{\prime} / E+c_{\mathrm{s}}^{2} k^{2}+3 \Omega_{\mathrm{m} 0}\left(c_{\mathrm{a}}^{2}-c_{\mathrm{s}}^{2}\right) \varpi
\end{aligned}
$$

where we have used Eq. (16) to replace derivatives of $\Psi$ with the observable $\Gamma$. So given observations of $\Gamma, \varpi$ and $E, \hat{Y}\left[c_{\mathrm{s}}^{2}, \Omega_{\mathrm{m} 0}\right]$ is a functional on that data depending on the parameters $\Omega_{\mathrm{m} 0}$ and $c_{\mathrm{s}}^{2}$ which outputs a function of scale and time.

However, we should stress that we can always find some $\hat{Y}$ given any data and given any choice of parameters. We have therefore thus far only obtained a model for $Y$ in the spirit of that provided by (19). It is a little more complicated, in that it depends not only on parameters equivalent to the $h_{i}$ 's, but also on observable data. If the observable $\Gamma$ shows no scale dependence, in principle we have a very simple model for $Y$, not dissimilar to the quasi-static one. If on the other hand, the observations do show scale dependence, $\hat{Y}$ could be a very complicated function of scale.

We must now test whether this model for $Y$ is consistent with the observations using the consistency relation (18), just as we did in the quasi-static case. This means that k-essence can only be a good description of the dark energy if there is no anisotropic stress observed and also

$$
\frac{\hat{Y}^{\prime}}{\hat{Y}}+\frac{2 \Omega_{\mathrm{m} 0} \hat{Y}}{P_{2}}=1+\Gamma,
$$

at every redshift and every scale. This consistency relation must be valid given just one global parameter $\Omega_{\mathrm{m} 0}$ and at each redshift the sound speed $c_{\mathrm{s}}^{2}$ and its derivative. Just as in the case of the quasi-static limit, at any one 
redshift, given measurements at four scales it is in principle enough to exclude such a model for $Y$ and therefore $\mathrm{k}$-essence as the mechanism for dark energy, assuming the existence of a perfect data set, as we already declared. ${ }^{3}$ Note that our approach is independent of the initial conditions.

Alternatively, we could have taken a time derivative of Eq. (21) and eliminated $\delta_{\mathrm{m}}$ using the available equations of motion. This gives a null test for k-essence purely in terms model-independent observables, but which involves $\Psi^{\prime \prime \prime}$. This procedure is exactly equivalent to using the consistency relation (25), which also involves an additional derivative.

If given the assumption of $\mathrm{k}$-essence, the consistency relation is satisfied at all redshifts, then the above measurements can be used to determine the value of the sound speed at each redshift observed. This allows a non-parametric determination of this physical property that in general is an arbitrary function of time.

It is worth mentioning the limit $c_{\mathrm{s}}^{2}=0[34,45]$, which is not quasi-static on any scale. In such a case, the dust and dark energy perturbations become indistinguishable and the entropy perturbation source in Eq. (21) disappears, yielding

$$
\Psi^{\prime \prime}+\left(4+\frac{E^{\prime}}{E}+3 \tilde{c}_{\mathrm{a}}^{2}\right) \Psi^{\prime}+\left(3+2 \frac{E^{\prime}}{E}+3 \tilde{c}_{\mathrm{a}}^{2}\right) \Psi=0 .
$$

where we have redefined the adiabatic sound speed to be that corresponding to the total EMT, $\tilde{c}_{\mathrm{a}}^{2} \equiv \dot{p}_{X} /\left(\dot{\rho}_{X}+\right.$ $\left.\dot{\rho}_{\mathrm{m}}\right)$, i.e. it is now a function purely of the background geometry. In this limit, there is no dependence on the unobservable $\delta_{\mathrm{m}}$ and all scale dependence disappears. A measurement of $Y$ is not possible in a fundamental sense, since there is no difference in the properties of the DE and the DM perturbations. The dark degeneracy is complete. The physics of the linear perturbations is effectively that of a single generalized dust collapsing on a background with some equation of state [34]. However, for such a model to be a valid description of the observations, the data must satisfy the constraint

$$
\Gamma^{\prime}+\left(\Gamma+3+2 \frac{E^{\prime}}{E}+3 \tilde{c}_{\mathrm{a}}^{2}\right)(\Gamma+1)+\frac{E^{\prime}}{E}=0
$$

at every redshift and every scale with no free parameters. This constraint reduces to exactly that of $\Lambda \mathrm{CDM}$ when the equation of state for the dark energy becomes -1 .

\footnotetext{
3 In fact, this would exclude any single perfect fluid as dark energy, since k-essence is equivalent to perfect-fluid hydrodynamics. Multiple perfect fluids or fluids with internal degrees of freedom have in general more complicated pressure perturbations, which would naively appear as more complicated scale dependence (e.g. [44]).
}

Models with kinetic gravity braiding (KGB) [46, 47] are the most general class of scalar-tensor theories with a single scalar which do not have a direct coupling to gravity and therefore do not have anisotropic stress despite being imperfect fluids [48]. The KGB equivalent of Eq. (21) would feature more scales, however the prescription for constraining this class of models would not differ from the k-essence example presented here. A null test for this class of theories would also involve third derivatives of the potential.

\section{Consistency with general Horndeski theories featuring anisotropic stress}

The most general second-order scalar-tensor theory is described by the Horndeski Lagrangian [36, 37]. The structure of the energy-momentum tensor for these theories is much more complex than for a model such as $\mathrm{k}$-essence. In particular, the Lagrangian features a nonminimal coupling to gravity. This means that the EMTs for any such models will feature, in general, anisotropic stress related to the perturbations of the scalar.

Horndeski models in the presence of dark matter have EMTs which have the following dependence on the perturbed fields when only linear perturbations are considered:

$$
\begin{aligned}
\delta T_{0}^{0} & \supset \dot{\delta \phi} \phi, \delta \phi, \delta_{\mathrm{m}}, \\
\delta T_{i}^{0} & \supset \dot{\delta \phi} \phi, \delta \phi, \theta_{\mathrm{m}}, \\
\delta T_{j}^{i}-1 / 3 \delta_{j}^{i} \delta T_{k}^{k} & \supset \sigma \delta \phi, \\
\delta T_{i}^{i} & \supset \ddot{\delta \phi} \phi, \dot{\delta \phi}, \delta \phi,
\end{aligned}
$$

in addition to dependence of all the components on the gravitational potentials $\Phi$ and $\Psi$ and their time derivatives. Here $\sigma$ is fully determined as a function of the Horndeski free functions. ${ }^{4}$ As compared to k-essence, we have the already mentioned anisotropic stress, which is always proportional to $\delta \phi$. The perturbation $\dot{\delta \phi}$ is present in the $(0 i)$ components of the EMT, while the pressure perturbation depends on $\ddot{\delta \phi}$. In addition to the above, we have the equation of motion for the scalar field, which is an equation for $\ddot{\delta \phi}$ in terms of all the other variables. The full expressions can be found in Ref. [38].

We can clearly see that the only way to suppress the anisotropic stress is to either make the coupling $\sigma$ very weak, i.e. effectively make the Horndeski terms nonminimally coupled to gravity irrelevant for the dynamics of dark energy, or to suppress the scalar perturbations, which is only possible if the scalar is very massive and not evolving, i.e. it just contributes vacuum energy [49].

\footnotetext{
${ }^{4}$ It is equivalent to the parameter $B_{7}$ in Ref. [38]. It is only nonzero when there is a direct coupling of the scalar to gravity in the action.
} 
Using the equation of motion for the scalar, we can eliminate $\ddot{\delta \phi}$ from the pressure equations. Then, using the Hamiltonian, momentum and anisotropy constraints [i.e. the $(00),(0 i)$ and $(i j)$ Einstein equations], we can eliminate three further variables: the scalar perturbations $\delta \phi, \dot{\delta \phi}$ and the dark-matter density $\delta_{\mathrm{m}}$. This is one extra variable as compared to models without anisotropic stress, where the anisotropy constraint equates the two potentials but is not dependent on the configuration of the dark energy degree of freedom. The remaining form for the $(i i)$ Einstein equation is very simple,

$$
\begin{aligned}
\sigma\left[\Phi^{\prime \prime}\right. & \left.+\alpha_{1} \Phi^{\prime}+\alpha_{2} \Psi^{\prime}+\left(\alpha_{3}+\alpha_{4} k^{2}\right) \Phi\right]+ \\
& +\left(\alpha_{5}+\alpha_{6} k^{2}\right)(\Phi+\Psi)=\sigma \alpha_{7} \Omega_{\mathrm{m}} k^{-2} \theta_{\mathrm{m}}
\end{aligned}
$$

where the parameters $\alpha_{i}$ are functions of time alone and are fully determined by the four free functions of the Horndeski Lagrangian. Their exact form will not be useful here.

We have kept the dependence on $\sigma$ explicit to show that in the limit of vanishing anisotropic stress, $\sigma \rightarrow 0$, Eq. (29) is not an alternative dynamical equation for the evolution of the potentials $\Phi$. To obtain the evolution equation, one would have to eliminate $\Psi$ for $\Phi$ and would obtain a very complicated equation with many scales embedded in its coefficients. One may worry that Eq. (29) provides a different version of this evolution equation than that obtained for theories with no anisotropic stress. This is not the case: switching off the anisotropic stress reduces Eq. (29) to an equation which enforces $\Phi=-\Psi$, thus providing no additional information.

Eq. (29) is exact at all scales where linear perturbation theory is valid. In Eq. (29), all the variables are modelindependent observables. Using the extra information from the anisotropic stress constraint, we have eliminated $\delta_{\mathrm{m}}$, which is not observable, and thus we can obtain a consistency relation between pure observables required by all Horndeski models with anisotropic stress,

$$
\begin{aligned}
& \eta \Gamma^{\prime}+\eta^{\prime \prime}+\Gamma\left(\eta \Gamma+2 \eta^{\prime}+\tilde{\alpha}_{1} \eta+\tilde{\alpha}_{2}\right)+ \\
& \quad+\tilde{\alpha}_{1} \eta^{\prime}+\tilde{\alpha}_{3} \eta+\tilde{\alpha}_{5}+k^{2}\left(\tilde{\alpha}_{4} \eta+\tilde{\alpha}_{6}\right)=\tilde{\alpha}_{7} \varpi .
\end{aligned}
$$

where we have redefined the $\alpha_{i}$ to absorb factors of $\sigma, E$ or $\Omega_{\mathrm{m}}$, or to combine coefficients into single variables.

A nice simple example of the relation (29) can be obtained in the case of the $f(R)$ class of dark energy models, which are a subclass of the Horndeski lagrangians and therefore feature much less freedom [50]. Following the algorithm described above, one obtains as the exact linear result

$$
\begin{aligned}
\Phi^{\prime \prime}-\Psi^{\prime} & +\left(4+\frac{E^{\prime}}{E}\right) \Phi^{\prime}+\frac{1}{3}\left(m_{\mathrm{C}}^{2}+2 k^{2}\right) \Phi+ \\
& +\frac{1}{3}\left(m_{\mathrm{C}}^{2}-6\left(2+\frac{E^{\prime}}{E}\right)+k^{2}\right) \Psi=0
\end{aligned}
$$

where $m_{\mathrm{C}}^{2} \equiv f_{, R} / 2 H^{2} f_{, R R}$ is the Compton mass of the scalar degree of freedom in the units of Hubble. We must have $m_{\mathrm{C}}^{2} \gg 1$ in order to satisfy Solar-System constraints [51]. At small enough scales, $k \gg m_{\mathrm{C}}$, we can recover the standard quasi-static result in $f(R)$ that $2 \Phi+\Psi=$ 0 . Again, we can express the above as a null test on observables that needs to be satisfied at all scales where linear theory is valid

$$
\begin{aligned}
\eta \Gamma^{\prime} & +\eta^{\prime \prime}+\Gamma\left(\eta \Gamma+2 \eta^{\prime}+5+\frac{E^{\prime}}{E}\right)+\left(4+\frac{E^{\prime}}{E}\right) \eta^{\prime}+ \\
& +\frac{m_{\mathrm{C}}^{2}}{3}(\eta-1)+6\left(2+\frac{E^{\prime}}{E}\right)+\frac{k^{2}}{3}(2 \eta-1)=0 .
\end{aligned}
$$

This relation has only one free parameter, $m_{\mathrm{C}}^{2}$, which can be freely adjusted at each redshift to fit the data. If this is not enough to satisfy the above test, then $f(R)$ is not a general enough theory to account for the observations.

The relation (30) [and (32)] is valid at all linear scales, without the need to involve the quasi-static limit or choose initial conditions. It is enough to make a detection of non-vanishing anisotropic stress at even just one scale and redshift to then require that the data satisfy (30). At any given redshift, the $\alpha_{i}$ coefficients are just numbers, independent of scale. The observables, $\Gamma, \eta$ and $\varpi$ are in principle complicated functions of scale and Eq. (30) is a non-linear scale-dependent function on these data. Performing observations for at least eight values of $k$ one can form an overconstrained system and rule out the Horndeski model, or confirm it while measuring its parameters $\alpha_{1-7}$, redshift by redshift without prior parametrization 5 .

\section{DISCUSSION AND CONCLUSIONS}

Dark matter is dark. This statement is obvious and yet the discussion of cosmological observations is usually framed as a discussion of the measurements of the darkmatter distribution. This is of course completely natural in the case where there are no dark energy perturbations, i.e. within the framework of $\Lambda \mathrm{CDM}$ cosmology. However, the moment that we relax this assumption, as we must do when investigating dark energy models different from a cosmological constant, this connection between measurements and the dark-matter distribution is no longer simple, and can to all intents and purposes disappear.

In this paper, we have stressed that the probes we hope to use to constrain general dark energy models depend

\footnotetext{
5 The parameters $\alpha_{1-7}$ are related to only four functions, in particular, those labelled as $w_{i}$ in Ref. [19]. Therefore, they are are in principle, reducible to a smaller set of parameters.
} 
on tracers which propagate on geodesics: light for weak lensing and galaxies for RSD. This allows us to map out the metric on our past lightcone (the potentials $\Phi$ and $\Psi$ ) through which these tracers fall freely. These are the real physical observables of the combination of the cosmological probes. The relation of these potentials to the darkmatter distribution is then a model-dependent statement, which just happens to be an identity for $\Lambda$ CDM.

Given this limitation, we should stress that the cosmological probes still can provide an enormous amount of information. Since we can reconstruct the potentials, the slip parameter $\eta$ of the matter/light Jordan-frame metric is a model-independent observable, given overlapping WL and RSD measurements. We do not need to parametrize it and we can answer the question of whether anisotropic stress is at all necessary, directly from observations, without any further modeling. This test has the power to immediately eliminate very large classes of models, whatever its result. If $\eta \neq 1$ at even one redshift and scale, then models without non-minimal coupling to gravity in the baryons' Jordan frame can be thrown away. If $\eta=1$ everywhere, then such couplings of dark energy to gravity could in principle still be there, but they are so small that they cannot at all influence the dynamics of the universe and therefore can be neglected. We have summarized the most important dynamical variables and their observability status in Table I.

We have also shown that the evolution rate of the potentials can be mapped out. On the other hand, the fact that we cannot observe the dark matter density amplitude means that the effective Newton's constant $Y$ is not an observable in a model-independent setting. Given these model-independent observables, we should refocus the predictions of perturbation theory in dark energy models to their impact on these gravitational potentials rather than on the dark matter, contrary to the dominant approach in the discussion today.

Nonetheless, we have demonstrated in section IV how to construct tests of very general classes of models of dark energy, even away from the frequently employed quasi-static limit. The distinct advantage of this approach is that rather than parametrizing the free functions of a class of models first, then evolving the predictions of these models using modified codes and simultaneously fitting to data, our method is capable of testing/constraining classes of models without any prior parametrization and without any assumptions on the initial conditions. These tests involve performing measurements of the observables at multiple scales, redshift by redshift (with the number of data points required determined purely by the number of free functions of time in the particular class of models). In order to carry out this test it is not necessary to modify codes for each class of models, but just to apply appropriate transformations to the observed data. In other words, our approach calls for exploring the space domain rather than the time domain of dark energy.

We have not addressed here the issue of whether such a method is indeed practical, delaying detailed forecasts to a separate follow-up work. A measurement of the slip parameter using Eq. (15) may not ever provide a better test for particular models of DE than the standard method. However, this test uniquely is model independent and it is not immediately obvious that the use of derivatives of data decreases information when we benefit by being able to construct a null test which must be satisfied at every scale and every redshift if $\Lambda \mathrm{CDM}$ is to not be excluded, especially if cosmic variance can be combatted.

The tests constructed beyond the quasi-static limit in section IV B do appear significantly less realistic. However, the standard methodology of parameterizing $\eta$ and $Y$ does not even address what might occur in situations where the quasi-static limit does not apply. Moreover, given the lack of well-motivated choices of potentials and initial conditions for DE models, eliminating a class of models, e.g. quintessence, as the mechanism for DE would significantly widen constraints since multiple potentials would have to be tested. It is thus unclear whether any other, more standard method would perform any better.

We have mostly assumed that there is no velocity bias between dark matter and the galaxies. Thus a measurement of galaxy peculiar velocities $\theta_{\text {gal }}$ via $\mathrm{RSD}$ is automatically a measurement of the dark-matter peculiar velocity $\theta_{\mathrm{m}}$. Statistical origins of the velocity bias notwithstanding [52], there could in principle be a nonvanishing bias between these two velocities. Indeed, this happens in any model in which there is a fifth force acting on dark matter, which does not couple to baryons or light, i.e. models with a violation of the equivalence principle such as coupled quintessence [33]. This fifth force is a new source of acceleration for dark-matter particles, causing their peculiar velocities to deviate from that of the galaxies. The effect of this is to introduce a (scale and time-dependent!) velocity bias. Our measurements of WL and RSD still map out the same metric potentials for our tracers, but these are not the complete potentials that the dark matter feels. Allowing for such deviations, we completely lose the connection between the dark matter growth rate and RSD of the galaxy power spectrum.

Summarizing the above, we can say that RSD and WL map out the Jordan-frame metric for galaxies and light, but provide no direct information on the Jordan frame of the underlying dark matter. In principle, one could use different tracers with different baryon fractions (cluster and galaxy power spectra) to map out the Jordan frames for these two classes of objects and obtain some information on the differences in the gravitational potentials they experience (in the spirit of e.g. [53]). Interestingly, galaxies are not just baryons, but are dynamically coupled to their dark-matter halos. This means that the Jordan-frame metric for the galaxies is not necessarily the same as the one for baryons, since the galaxies will at least partly feel the fifth force acting on dark-matter. 


\begin{tabular}{|c|c|c|c|c|}
\hline Class & Variable & Key Relation & Obs.? Eq. & Comment \\
\hline \multirow[t]{4}{*}{ Measurements } & $A$ & $\equiv \delta_{\text {gal }}=b \delta_{\mathrm{m}}$ & $\checkmark \quad(7)$ & Bias not measurable absent DE model \\
\hline & $R$ & $\equiv-\theta_{\text {gal }} / H$ & $\checkmark \quad(7)$ & RSD measure galaxy velocity \\
\hline & $L$ & $\equiv \frac{2 k^{2} E^{2}}{3(1+z)^{3}}(\Phi-\Psi)$ & $\checkmark \quad(7)$ & WL shear probes lensing potential \\
\hline & $E$ & $\equiv H / H_{0}$ & $\checkmark$ & $H_{0}, \Omega_{\mathrm{m} 0}$ not observable without DE model ${ }^{\mathrm{a}}$ \\
\hline \multirow{3}{*}{$\begin{array}{l}\text { Primary } \\
\text { observables }\end{array}$} & $P_{1}=\beta$ & $\equiv R / A=f / b$ & $\checkmark \quad(8)$ & \\
\hline & $P_{2}=E_{G}$ & $\equiv L / R=(1+\eta) / \varpi$ & $\checkmark \quad(8)$ & \\
\hline & $P_{3}$ & $\equiv R^{\prime} / R=f+f^{\prime} / f$ & $\checkmark \quad(8)$ & Only this function of $f$ is observable \\
\hline \multirow{4}{*}{$\begin{array}{l}\text { Physical } \\
\text { variables }\end{array}$} & $\Psi$ & $R^{\prime}+R\left(2+E^{\prime} / E\right)$ & $\checkmark(14)$ & Extract from RSD \\
\hline & $\Phi$ & $\frac{3(1+z)^{3}}{2 k^{2} E^{2}} L+\Psi$ & $\checkmark$ & Extract from WL tomography \\
\hline & $\delta_{\mathrm{m}}$ & $\delta_{\mathrm{m}}=\delta_{\text {gal }} / b$ & & Unknown without knowing bias \\
\hline & $\theta_{\mathrm{m}} / H_{0}$ & $\theta_{\mathrm{m}}=\theta_{\text {gal }}$ & $\checkmark$ & Observable only given equivalence principle \\
\hline$D E$ & $\eta$ & $\equiv-\Phi / \Psi$ & $\checkmark \quad(3)$ & $\eta \neq 1 \Rightarrow$ coupling DE/gravity \\
\hline Configuration & $\Gamma$ & $\equiv \Psi^{\prime} / \Psi$ & $\checkmark(16)$ & Observable prediction of DE models \\
\hline \multirow[t]{3}{*}{ Variables } & $Y$ & $\equiv-2 k^{2} \Psi / 3 \Omega_{\mathrm{m}} \delta_{\mathrm{m}}$ & $(3)$ & $\delta_{\mathrm{m}}$ unknown; must satisfy relation $(18)$ \\
\hline & $f$ & $\equiv-\theta_{\mathrm{m}} / H \delta_{\mathrm{m}} \approx \delta_{\mathrm{m}}^{\prime} / \delta_{\mathrm{m}}$ & $(3)$ & $\delta_{\mathrm{m}}$ unknown \\
\hline & $\varpi$ & $\equiv f / \Omega_{\mathrm{m} 0} Y$ & $\checkmark \quad(6)$ & Relates DM velocity to potential \\
\hline
\end{tabular}

a See Ref. [19] for a detailed discussion of the observability of the background.

Table I. Summary of variables used in this paper. Tshose variables marked with a checkmark as observable can be measured as a function of redshift and scale without an assumption of a particular DE model. Measurement of $\delta_{\mathrm{m}}$ requires the knowledge of bias, which cannot be measured or modeled without the knowledge of the DE model. The variables such as $Y$ and $f$ typically used to describe the DE configuration are therefore not observable, but predictions can be reformulated to maximally exploit those variables which are observable (see section IV B).

Another interesting implication of our approach is that potential non-linearities of dark energy, which can appear on very different scales to those in dark matter as a result of the generic existence of screening mechanisms, are not deadly to the measurements of the linear potentials $\Phi$ and $\Psi$. For tracers to move on geodesics determined by these potentials, their gradients must remain small, i.e. the total EMT perturbations must be linear. So even if the DE contribution is non-linear, it is enough for the dark-matter density to be dominant and its perturbation linear for the tracers to continue to move on geodesics described by $\Phi$ and $\Psi$. However, since there will no doubt be non-linear contributions to the potentials, both from the DM and the DE perturbations, this should imply that measurements of higher-order correlation functions should be sensitive to the extra non-linearity as compared to the expected $\Lambda \mathrm{CDM}$ result.

Let us pre-emptively address a few potential criticisms: our results and approach is driven by the limitations in our ability to determine the galaxy bias $b$ as well as the normalization $\sigma_{8}$ and scale dependence of the darkmatter perturbations $\delta_{\mathrm{m}}$. There exist many, increasingly better, measurements of these quantities e.g. [54-58]. However, they all depend in a fundamental way on assumptions necessarily true only in $\Lambda \mathrm{CDM}$ and therefore we cannot use them.

Although non-linearities can be used to constrain the bias through the galaxy bispectrum [58], this is a modeldependent statement since screening mechanisms present in most dark energy models alter the expectations. Otherwise, a measurement of bias is usually obtained as a result of measuring $\sigma_{8}$, which is done using either cluster counts and/or weak lensing around clusters. As we have already pointed out, weak lensing measures the lensing potential rather than the DM distribution, i.e. includes a dependence on the DE perturbations. To extract information from cluster counts, their mass is obtained from their gas temperature - which gives information on the Newtonian potential $\Psi$ and not on the DM mass - and spherical collapse or N-body simulations are used, both of which depend deeply on the model for dark energy, see e.g. Refs [47, 59, 60]. All of these issues can in principle be appropriately modeled, but this must be done on a model-by-model basis. Without this calculation, we cannot interpret 
the $\Lambda$ CDM measurement of $\sigma_{8}$ as a parameter valid for other models of dark energy.

The tests that we have constructed in section IV B depend on eliminating variables through the use of the constraints present in the Einstein equations. Given a class of models for $\mathrm{DE}$, one can replace dynamical variables with combinations of observables. Given the assumption that the DE is a single degree of freedom, that the equivalence principle be satisfied and a detection of nonvanishing anisotropic stress, we have enough information to eliminate all non-observable quantities from the evolution equations and thus are able to form a null test for the most general class of scalar-tensor models directly on the observable data.

When there is no anisotropic stress then no such complete constraint can be formed: the dependence on the unobservable $\delta_{\mathrm{m}}$ remains. In that case, the best that can be done is to obtain a measurement of the effective Newton's constant $Y$ on the assumption that a particular class of models of dark energy describes the Universe and then use a consistency relation for $Y$ to determine whether the assumption was consistent with the data. This is a somewhat more complicated exercise, which we have demonstrated for perfect-fluid k-essence models, but would also apply to imperfect-fluid models featuring kinetic gravity braiding $[37,47,48]$.

Tests of the type we have shown can in principle be constructed for any other class of DE models. Indeed, we would like to argue that one should think of the parametrized or effective approaches, such as those of Refs $[14,16-18]$, as providing the dynamics of the dark energy which can be rewritten in terms of evolution equations for the potentials. Provided they are written in terms of parameters that on cosmological solutions are only functions of time, a test such as those presented in section IV would allow for putting constraints on these parameters, or indeed would exclude setups which posses insufficient operators to describe the data fully.

An alternative way of thinking about these tests is that, using the measurement of $\Phi$ and $\Psi$, we are essentially reconstructing the components of an effective combined EMT for DM and DE [61]. Now, given a class of models, we can extract relations between the configuration of the degrees of freedom and the fluid variables, and therefore between the fluid variables themselves, i.e. anisotropic stress, pressure, energy density. In general this is difficult, but in some classes of models it can be done, e.g. [13, 15]. However, it should be intuitively clear that if the dark energy has more degrees of freedom, only the adiabatic modes will influence the potentials. Internal modes, since they do not affect the gravitational field of the DE, would only be constrainable through their impact on the time evolution of the dark energy. In particular, one could also form null tests on observables, but these would involve taking additional derivatives and eliminating the internal degrees of freedom through the appropriate equations of motion: essentially two extra derivatives of the potentials would need to be taken for every additional degree of freedom in the DE model class.

We would like to stress that our method depends on taking derivatives of the data. Since for the purposes of this paper we are working in the idealized case of sufficiently good data, we have not addressed here the feasibility of this procedure in a realistic situation. We leave this for future work.

We have also used a number of simplifying assumptions for the relation of measurements to the potentials. For example, the Kaiser formula (11), assumes not only linearity, but also a flat sky and ignores near-horizon effects. As shown by e.g. [31], the correction involve contributions from, for example, weak lensing, which would pollute the determination of the peculiar velocities of galaxies from RSDs. Indeed there are also near horizon corrections to the weak lensing, e.g. [28]. Since our aim is to exploit large-scale data fully, taking into account such corrections is a natural extension of this work.

\section{ACKNOWLEDGMENTS}

We would like to thank Eugeny Babichev, Nicolas Busca, Christos Charmoussis, Valerio Marra, Federico Piazza, Glenn Starkman and Alexander Vikman for helpful comments and suggestions. The work of L.A. and I.S. is supported by the DFG through TRR33 "The Dark Universe". M.K. acknowledges funding by the Swiss National Science Foundation. M.M. is supported by CNPqBrazil. I.D.S. acknowledges STFC for financial support.
1] A. Shafieloo and C. Clarkson, "Model independent tests of the standard cosmological model,"

Phys.Rev. D81 (2010) 083537, arXiv:0911.4858 [astro-ph.CO].

[2] A. Shafieloo and E. V. Linder, "Cosmographic Degeneracy," Phys.Rev. D84 (2011) 063519, arXiv:1107.1033 [astro-ph.CO].

[3] D. Huterer and G. Starkman, "Parameterization of dark-energy properties: A Principal-component approach," Phys.Rev.Lett. 90 (2003) 031301, arXiv: astro-ph/0207517 [astro-ph].

[4] R. R. Caldwell and M. Doran, "Dark-energy evolution across the cosmological-constant boundary," Phys.Rev. D72 (2005) 043527, arXiv:astro-ph/0501104 [astro-ph].

[5] E. V. Linder, "Cosmic growth history and expansion history," Phys.Rev. D72 (2005) 043529, arXiv:astro-ph/0507263 [astro-ph]. 
[6] W. Hu and I. Sawicki, "A Parameterized Post-Friedmann Framework for Modified Gravity," Phys.Rev. D76 (2007) 104043, arXiv:0708.1190 [astro-ph].

[7] M. A. Amin, R. V. Wagoner, and R. D. Blandford, "A sub-horizon framework for probing the relationship between the cosmological matter distribution and metric perturbations," Mon.Not.Roy.Astron.Soc. 390 (2008) 131-142, arXiv:0708.1793 [astro-ph].

[8] L. Amendola, M. Kunz, and D. Sapone, "Measuring the dark side (with weak lensing)," JCAP 0804 (2008) 013, arXiv:0704.2421 [astro-ph].

[9] R. Bean and M. Tangmatitham, "Current constraints on the cosmic growth history,"

Phys.Rev. D81 (2010) 083534, arXiv: 1002.4197 [astro-ph.CO].

[10] L. Pogosian, A. Silvestri, K. Koyama, and G.-B. Zhao, "How to optimally parametrize deviations from General Relativity in the evolution of cosmological perturbations?," Phys.Rev. D81 (2010) 104023, arXiv:1002.2382 [astro-ph.CO].

[11] M. Kunz, "The phenomenological approach to modeling the dark energy,"

Comptes Rendus Physique 13 (2012) 539-565, arXiv: 1204.5482 [astro-ph.CO].

[12] E. Bertschinger, "On the Growth of Perturbations as a Test of Dark Energy," Astrophys.J. 648 (2006) 797-806, arXiv:astro-ph/0604485 [astro-ph].

[13] R. A. Battye and J. A. Pearson, "Effective action approach to cosmological perturbations in dark energy and modified gravity," JCAP 1207 (2012) 019, arXiv:1203.0398 [hep-th].

[14] T. Baker, P. G. Ferreira, and C. Skordis, "The Parameterized Post-Friedmann Framework for Theories of Modified Gravity: Concepts, Formalism and Examples," Phys.Rev. D87 (2013) 024015, arXiv:1209.2117 [astro-ph.CO].

[15] I. Sawicki, I. D. Saltas, L. Amendola, and M. Kunz, "Consistent perturbations in an imperfect fluid," JCAP 1301 (2013) 004, arXiv:1208.4855 [astro-ph.CO].

[16] G. Gubitosi, F. Piazza, and F. Vernizzi, "The Effective Field Theory of Dark Energy," JCAP 1302 (2013) 032, arXiv:1210.0201 [hep-th].

[17] J. K. Bloomfield, E. E. Flanagan, M. Park, and S. Watson, "Dark Energy or Modified Gravity? An Effective Field Theory Approach," arXiv:1211.7054 [astro-ph.CO].

[18] J. Gleyzes, D. Langlois, F. Piazza, and F. Vernizzi, "Essential Building Blocks of Dark Energy," arXiv:1304.4840 [hep-th].

[19] L. Amendola, M. Kunz, M. Motta, I. D. Saltas, and I. Sawicki, "Observables and unobservables in dark energy cosmologies," Phys.Rev. D87 (2013) 023501, arXiv:1210.0439 [astro-ph.CO].

[20] U. Seljak, "Extracting primordial non-gaussianity without cosmic variance,"

Phys.Rev.Lett. 102 (2009) 021302, arXiv:0807.1770 [astro-ph].

[21] M. Kunz, "The dark degeneracy: On the number and nature of dark components," Phys.Rev. D80 (2009) 123001, arXiv:astro-ph/0702615 [astro-ph].

[22] A. G. Riess, L. Macri, S. Casertano, H. Lampeitl, H. C.
Ferguson, et al., "A 3\% Solution: Determination of the Hubble Constant with the Hubble Space Telescope and Wide Field Camera 3," Astrophys.J. 730 (2011) 119, arXiv:1103.2976 [astro-ph.CO].

[23] V. Marra, L. Amendola, I. Sawicki, and W. Valkenburg, "Cosmic variance and the measurement of the local Hubble parameter," arXiv:1303.3121 [astro-ph.CO] .

[24] P. Zhang, M. Liguori, R. Bean, and S. Dodelson, "Probing Gravity at Cosmological Scales by Measurements which Test the Relationship between Gravitational Lensing and Matter Overdensity," Phys.Rev.Lett. 99 (2007) 141302, arXiv:0704.1932 [astro-ph].

[25] W. Hu, "Power spectrum tomography with weak lensing," Astrophys.J. 522 (1999) L21-L24, arXiv:astro-ph/9904153 [astro-ph].

[26] N. Kaiser, "Clustering in real space and in redshift space," Mon.Not.Roy.Astron.Soc. 227 (1987) 1-27.

[27] R. Scoccimarro, H. Couchman, and J. A. Frieman, "The Bispectrum as a signature of gravitational instability in redshift-space," Astrophys.J. 517 (1999) 531-540, arXiv:astro-ph/9808305 [astro-ph].

[28] F. Bernardeau, C. Bonvin, and F. Vernizzi, "Full-sky lensing shear at second order," Phys.Rev. D81 (2010) 083002, arXiv:0911.2244 [astro-ph.CO].

[29] J. Yoo, A. L. Fitzpatrick, and M. Zaldarriaga, "A New Perspective on Galaxy Clustering as a Cosmological Probe: General Relativistic Effects,"

Phys.Rev. D80 (2009) 083514, arXiv:0907.0707 [astro-ph.CO].

[30] A. Challinor and A. Lewis, "The linear power spectrum of observed source number counts," Phys.Rev. D84 (2011) 043516, arXiv:1105.5292 [astro-ph.CO].

[31] C. Bonvin and R. Durrer, "What galaxy surveys really measure," Phys.Rev. D84 (2011) 063505, arXiv:1105.5280 [astro-ph.CO].

[32] J. Yoo, N. Hamaus, U. Seljak, and M. Zaldarriaga, "Going beyond the Kaiser redshift-space distortion formula: a full general relativistic account of the effects and their detectability in galaxy clustering,"

Phys.Rev. D86 (2012) 063514, arXiv:1206.5809 [astro-ph.CO].

[33] L. Amendola, "Coupled quintessence," Phys.Rev. D62 (2000) 043511 arXiv: astro-ph/9908023 [astro-ph].

[34] E. A. Lim, I. Sawicki, and A. Vikman, "Dust of Dark Energy," JCAP 1005 (2010) 012, arXiv:1003.5751 [astro-ph.CO].

[35] A. Silvestri, L. Pogosian, and R. V. Buniy, "A practical approach to cosmological perturbations in modified gravity," arXiv:1302.1193 [astro-ph.CO].

[36] G. W. Horndeski, "Second-order scalar-tensor field equations in a four-dimensional space," Int.J.Th.Phys. 10 (1974) 363-384.

[37] C. Deffayet, X. Gao, D. Steer, and G. Zahariade, "From k-essence to generalised Galileons," Phys.Rev. D84 (2011) 064039, arXiv:1103.3260 [hep-th].

[38] A. De Felice, T. Kobayashi, and S. Tsujikawa, "Effective gravitational couplings for cosmological perturbations in the most general scalar-tensor theories with second-order field equations," 
Phys.Lett. B706 (2011) 123-133,

arXiv: 1108.4242 [gr-qc].

[39] C. Armendariz-Picon, T. Damour, and V. F. Mukhanov, "k - inflation," Phys.Lett. B458 (1999) 209-218, arXiv:hep-th/9904075 [hep-th].

[40] C. Armendariz-Picon, V. F. Mukhanov, and P. J. Steinhardt, "A Dynamical solution to the problem of a small cosmological constant and late time cosmic acceleration," Phys.Rev.Lett. 85 (2000) 4438-4441, arXiv:astro-ph/0004134 [astro-ph].

[41] C. Armendariz-Picon, V. F. Mukhanov, and P. J. Steinhardt, "Essentials of k essence," Phys.Rev. D63 (2001) 103510, arXiv:astro-ph/0006373 [astro-ph].

[42] J. Garriga and V. F. Mukhanov, "Perturbations in k-inflation," Phys.Lett. B458 (1999) 219-225, arXiv:hep-th/9904176 [hep-th].

[43] V. Mukhanov, Physical Foundations of Cosmology. Cambridge Univ. Press, Cambridge.

[44] M. Kunz and D. Sapone, "Crossing the Phantom Divide," Phys.Rev. D74 (2006) 123503, arXiv:astro-ph/0609040 [astro-ph] .

[45] P. Creminelli, G. D'Amico, J. Norena, L. Senatore, and F. Vernizzi, "Spherical collapse in quintessence models with zero speed of sound," JCAP 1003 (2010) 027, arXiv:0911.2701 [astro-ph.CO].

[46] C. Deffayet, O. Pujolas, I. Sawicki, and A. Vikman, "Imperfect Dark Energy from Kinetic Gravity Braiding," JCAP 1010 (2010) 026, arXiv: 1008.0048 [hep-th].

[47] R. Kimura and K. Yamamoto, "Large Scale Structures in Kinetic Gravity Braiding Model That Can Be Unbraided," JCAP 1104 (2011) 025, arXiv:1011.2006 [astro-ph.CO].

[48] O. Pujolas, I. Sawicki, and A. Vikman, "The Imperfect Fluid behind Kinetic Gravity Braiding," JHEP 1111 (2011) 156, arXiv:1103.5360 [hep-th].

[49] I. D. Saltas and M. Kunz, "Anisotropic stress and stability in modified gravity models," Phys.Rev. D83 (2011) 064042, arXiv:1012.3171 [gr-qc].

[50] A. De Felice and S. Tsujikawa, " $\mathrm{f}(\mathrm{R})$ theories," Living Rev.Rel. 13 (2010) 3, 1002.4928.

[51] W. Hu and I. Sawicki, "Models of $f(R)$ Cosmic Acceleration that Evade Solar-System Tests,"
Phys.Rev. D76 (2007) 064004,

arXiv:0705.1158 [astro-ph].

[52] V. Desjacques and R. K. Sheth, "Redshift space correlations and scale-dependent stochastic biasing of density peaks," Phys.Rev. D81 (2010) 023526, arXiv:0909.4544 [astro-ph.CO].

[53] L. Hui and A. Nicolis, "Proposal for an Observational Test of the Vainshtein Mechanism," Phys.Rev.Lett. 109 (2012) 051304, arXiv:1201.1508 [astro-ph.CO].

[54] E. Rozo et al., "Cosmological Constraints from the SDSS maxBCG Cluster Catalog," Astrophys.J. 708 (2010) 645-660, arXiv:0902.3702 [astro-ph.C0].

[55] J. L. Tinker, E. S. Sheldon, R. H. Wechsler, M. R. Becker, E. Rozo, et al., "Cosmological Constraints from Galaxy Clustering and the Mass-to-Number Ratio of Galaxy Clusters," Astrophys.J. 745 (2012) 16, arXiv:1104.1635 [astro-ph.CO].

[56] R. Tojeiro, W. Percival, J. Brinkmann, J. Brownstein, D. Eisenstein, et al., "The clustering of galaxies in the SDSS-III Baryon Oscillation Spectroscopic Survey: measuring structure growth using passive galaxies," Mon.Not.Roy.Astron.Soc. 424 (2012) 2339-2344, arXiv:1203.6565 [astro-ph.CO].

[57] R. Mandelbaum, A. Slosar, T. Baldauf, U. Seljak, C. M. Hirata, et al., "Cosmological parameter constraints from galaxy-galaxy lensing and galaxy clustering with the SDSS DR7," arXiv:1207.1120 [astro-ph.CO].

[58] WiggleZ Collaboration Collaboration, F. A. Marin et al., "The WiggleZ Dark Energy Survey: constraining galaxy bias and cosmic growth with 3-point correlation functions," arXiv:1303.6644 [astro-ph.CO].

[59] F. Schmidt, W. Hu, and M. Lima, "Spherical Collapse and the Halo Model in Braneworld Gravity," Phys.Rev. D81 (2010) 063005, arXiv:0911.5178 [astro-ph.CO].

[60] A. Borisov, B. Jain, and P. Zhang, "Spherical Collapse in f(R) Gravity," Phys.Rev. D85 (2012) 063518, arXiv:1102.4839 [astro-ph.CO].

[61] G. Ballesteros, L. Hollenstein, R. K. Jain, and M. Kunz, "Nonlinear cosmological consistency relations and effective matter stresses," JCAP 1205 (2012) 038, arXiv:1112.4837 [astro-ph.CO]. 